\title{
Analysis of the complementary energy losses of a high temperature gas to gas heat exchanger based on a solid intermediate medium
}

\author{
L. A. Catalano, R. Amirante, P. Tamburrano \& S. Copertino \\ Polytechnic of Bari, Italy
}

\begin{abstract}
This paper focuses on the analysis of the complementary energy losses of a gas to gas heat exchanger using an intermediate medium with high thermal capacity to transfer heat between two gas streams at different pressure. In fact, such a heat exchanger requires two mechanical devices, namely a pressurization system capable of transferring the particles from a low pressure to a high pressure environment, and a depressurization system, for the inverse procedure. The operating procedures of these two key components are presented in detail and a simple zero-dimensional approach is also proposed to model both systems. A numerical analysis, based on the same zero-dimensional model, is performed in order to evaluate all energy losses related to the pressurization and depressurization procedures. A further energy loss is analysed, in particular the one related to the energy absorbed by the conveyor employed to deliver back the particles from the bottom to the top of the plant in order to realize a continuous operation mode.
\end{abstract}

Keywords: gas turbines, external combustion, heat exchanger, particle pressurization.

\section{Introduction}

The application of externally fired gas turbines for power generation would offer the possibility of burning "dirty" fuels, like coal or orimulsion or biomass, since the combustion gases are not in direct contact with the moving parts of the machine in the external combustion operation mode. On the contrary, modern energy conversion technologies like internal combustion gas turbines demand the use of clean fuels, which are significantly more expensive. Even if externally 
fired gas turbines would have the advantage of burning cheaper fuels, their development and application is limited by the intrinsic difficulties in realizing gas to gas heat exchangers working at very high temperature and characterized by high thermal efficiency and by a limited pressure drop. As well, designing and manufacturing high-efficiency heat exchangers is also a limiting factor in the development of gas turbines employing the heat recovery Joule-Braxton cycle [1-4].

The so-called "pebble-bed" devices represent an alternative to the traditional gas to gas heat exchangers: they make use of an intermediate medium (a granular ceramic or metallic material) first to recover and then to release heat from one flow to another; this has been achieved in an alternate operation mode by using two tanks with large alumina balls where hot and cold air flow alternatively [5]. To authors' knowledge, there are only two examples of pebble-bed devices working in a continuous operation mode, namely, two patents of the 1960s [6, 7]. Both of them are made of two heat exchange modules: the particles are heated up in the first one and then transfer the heat to the cold flow in the second one; at the same time, the particles are cooled and, thus, are enabled to repeat the process. The two devices mainly differ in the way the particles cross the air flows: one of them [6] is completely gravity-based (particles fall after their injection in the gas flow), while a series of tilted rotating plates drives their path in the other one [7]. Pebble-bed heat exchangers operating in a continuous mode could have some advantages in comparison with traditional ones: high efficiency, low pressure drops, and relatively simple construction. However, both cited proposals did not include detailed fluid-dynamic studies aiming at optimizing the particle trajectories; moreover, they did not mention how hot particles can be injected in the high pressure gas flow without crushing them.

Some of the authors have recently proposed and optimized [8-10] an innovative Immersed Particle Heat Exchanger, which employs an intermediate medium with high thermal capacity: with reference to Figure 1, small alumina particles fall in a column where hot gas, coming from a separate combustion chamber (external combustion gas turbine) or from turbine outlet (heat recovery cycle), flows from the bottom to the top; the warmed up particles are then collected at the bottom of the column and inserted at the top of a second column where they transfer the accumulated heat to a counter-flowing cold gas. Cold particles are collected at the bottom of the second module and delivered back at the top of the plant by the conveyor. The potential of such heat exchange mode has been demonstrated both theoretically and experimentally: an effective onedimensional model, which allows us to compute the column lengths required for achieving the heat exchanger design efficiency, was proposed and validated by means of a test bench, reproducing the upper half of the proposed Immersed Particle Heat Exchanger [8]. It is noteworthy that, due to the small particle size, conduction inside the intermediate medium can be neglected, as demonstrated in [10] by means of DNS simulations. The experimental facility was also equipped with a second vertical pipe, used to demonstrate that very fine particles, which could damage turbine blades in a real plant, can be completely eliminated by means of centrifugation $[8,9]$. In addition, a tri-dimensional CFD 
model, capable of recognizing all 3D geometrical details, was developed and used to optimize the main geometrical parameters affecting the heat exchange effectiveness [9].

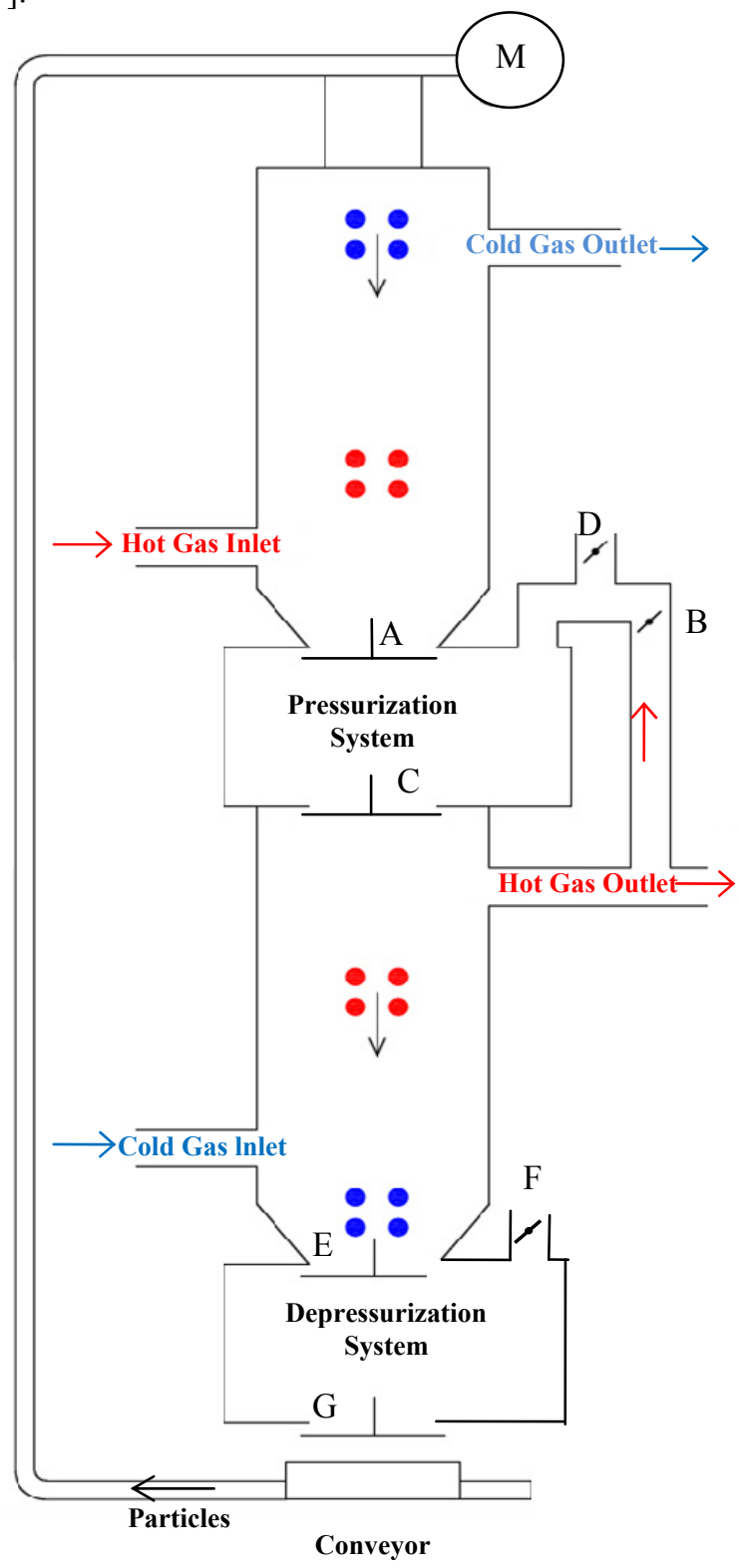

Figure 1: Immersed Particle Heat Exchanger. 
A mechanical system interposed between the two columns is needed to pressurize the solid intermediate medium; afterwards, the particles can fall in the second column thanks to gravity. As well, a second mechanical system must be placed below the bottom column in order to depressurize and transfer the particles into a pneumatic conveyor, capable of delivering back the particles to the top of the plant for a continuous operation mode. Such devices must operate without crushing the particles, both to maintain their size constant and to avoid dust [8].

The development of the pressurization and depressurization systems is proposed in this paper: their operating principles are proposed in the next section; in section 3, a simple zero-dimensional model is employed for analysing the main energy losses, occurring during the operating procedure of both the pressurization system and the depressurization system. Finally, a further energy loss, due to the energy absorbed by the conveyor, is evaluated with respect to the overall thermal energy exchanged between the particles and the gas in the bottom column.

\section{Operating principles of the pressurization and of the depressurization systems}

As shown in Figure 1, the pressurization system proposed in this paper is mainly composed of a pressurization tank interposed between the two heat exchange modules, one pipe connecting the tank with the bottom duct and four control valves (denoted with A, B, C, D in Fig.1). The opening and closing of the control valves need to be regulated by a control system.

One pressurization cycle consists of the following four steps:

1. Tank filling: at the beginning of the cycle, the four valves A, B, C and D are in closed position: the first phase starts when the valve A opens and the solid particles start falling into the pressurization tank.

2. Pressurization: once the particles are in the tank, the valve A closes and the pressurization phase starts by opening the pressurization valve B: a small amount of compressed gas moves from the bottom duct to the central tank. As a result, the pressure in the tank increases until attaining the pressure in the bottom column.

3. Particle discharge (from the pressurization tank to the bottom column): this phase starts when the valve $C$ opens and the solid particles can fall down for gravity from the pressurization tank into the bottom duct.

4. Gas discharge: when the particles have been discharged from the central tank, valves $\mathrm{B}$ and $\mathrm{C}$ can be closed; the compressed gas remaining in the tank needs to be ejected in order to lower pressure to the atmospheric value and to start a new cycle. Thus, valve $\mathrm{D}$ is opened.

The depressurization system has a similar operation mode: it is composed of one depressurization tank placed below the bottom column and three control valves (denoted with E, F, G in Figure 1). Even in this case, the opening and closing of the control valves must automatically be regulated by the implemented 
control system. The following three steps are performed during one depressurization cycle:

1. Tank filling: the first phase starts when the valve $E$ opens and the solid particles can fall into the depressurization tank: a small amount of compressed gas moves from the bottom column to the depressurization tank together with the particles. During this phase, valves $F$ and $G$ are in the closed position.

2. Depressurization: after the particles have entered the bottom tank, the valve $\mathrm{E}$ closes and the depressurization phase begins by opening the depressurization valve $\mathrm{F}$ : the compressed gas, which occupies the interstices of the particles, is ejected from the bottom tank to the external environment. As a result, the pressure in the bottom tank decreases to the atmospheric pressure.

3. Particle discharge (from the depressurization tank to the conveyor): after the valve $F$ has been closed, the valve $G$ opens and the solid particles can fall down from the depressurization tank into the particle conveyor.

\section{Analysis of energy losses}

\subsection{Energy loss due to the lost compressed gas}

The compressed gas ejected into the external environment represents an energy loss, which is investigated in this section by means of the quantification of the overall percentage of the gas flow rate which is lost.

The mass flow rate of compressed gas discharged into the external environment, $\dot{m}_{\text {lost }}$, can be split as:

$$
\dot{m}_{\text {lost }}=\dot{m}_{\text {lost }}^{P}+\dot{m}_{\text {lost }}^{D}
$$

where $\dot{m}_{\text {lost }}^{P}$ and $\dot{m}_{\text {lost }}^{D}$ are the mass flow rate of gas discharged during one pressurization cycle and during one depressurization cycle, respectively.

With regard to the pressurization system, the connection between the bottom column and the central tank is kept open by the pressurization valve (valve B in Figure 1) during the discharge of the solid particles from the central tank into the bottom column. At the end of this phase, which occurs when all solid particles have been transferred from the pressurization tank into the bottom column, and the valve $\mathrm{C}$ and $\mathrm{B}$ are closed, the pressurization tank is full of compressed gas. In order to repeat the pressurization cycle, this gas must be ejected to the outside and this is achieved during the last phase of the cycle, by opening the valve $D$. The corresponding mass flow rate of compressed gas discharged into the external environment, $\dot{m}_{\text {lost }}^{P}$, can be expressed as follows:

$$
\dot{m}_{\text {lost }}^{P}=\frac{m_{\text {lost }}^{P}}{t_{c}^{P}}
$$

where $t_{c}^{P}$ denotes the duration of each pressurization cycle and $m_{\text {lost }}^{P}$ is the mass of compressed gas lost in one pressurization cycle: with the worst hypothesis, this amount can be considered equal to the entire mass of gas in the central tank 
immediately before the opening of the valve D. Therefore, with this hypothesis and denoting with $\rho_{g}^{\text {out }}$ the gas density at the outlet of the bottom duct and with $V_{t}^{P}$ the volume of the central tank, Equation (2) can be written as:

$$
\dot{m}_{\text {lost }}^{P}=\frac{\rho_{g}^{\text {out }} v_{t}^{P}}{t_{c}^{P}}
$$

With regard to $t_{c}^{P}$, it can be split as:

$$
t_{c}^{P}=2 t_{d}^{P}+t_{x}^{P}
$$

where $t_{d}^{P}$ is the time interval of the particle discharge phase, which can be considered equal to the time interval spent for the particle discharge phase if the valves $\mathrm{A}$ and $\mathrm{C}$ have the same size [11], and $t_{x}^{P}$ amounts the time intervals spent for the pressurization, for the gas discharge and for the valves actuation.

The particles are discharged intermittently from the central tank into the bottom column, indeed the mass flow rate of particles varies with time from a constant value (when the valve $\mathrm{C}$ is open) to zero (in the remaining period of the pressurization cycle). Since the Immersed Particle Heat Exchanger must operate continuously in a real plant, an accumulation grid must be inserted below the pressurization system, in order to achieve a constant mass flow rate of particles in the bottom column. Denoting with $\overline{\dot{m}}_{p}$ the average mass flow rate of particles discharged from the pressurization tank into the bottom column, $\overline{\dot{m}}_{p}$ can be related to the tank volume as follows:

$$
\overline{\dot{m}}_{p}=\frac{\rho_{p} v_{t}^{P}}{t_{c}{ }^{P}}
$$

It is noteworthy that $\overline{\dot{m}}_{p}$ must be equal to the mass flow rate required for heating the compressed gas in the bottom column. Since thermal capacities of particles and of gas must be equal for a counterflow heat exchanger, $\dot{\dot{m}}_{p}$ must be computed as:

$$
\overline{\dot{m}}_{p}=\frac{c_{p, g} \dot{m}_{g}}{c_{p}}
$$

In equation (6), $c_{p, g}$ is the gas specific heat (supposed constant), $c_{p}$ is the particle specific heat and $\dot{m}_{g}$ is the gas mass flow rate flowing through the bottom column.

Combining Equations (5) and (6), one obtains Equation (7), which expresses the volume of the pressurization tank depending on the gas mass flow rate, the phisical properties of the particles, and the cycle time (or, equivalently, the number of cycles per seconds, indicated with $n^{P}$ ):

$$
V_{t}^{P}=\frac{c_{p, g} \dot{m}_{g}}{\rho_{p} c_{p}} t_{c}^{P}=\frac{c_{p, g} \dot{m}_{g}}{\rho_{p} c_{p}} \frac{1}{n^{P}}
$$

With regard to the depressurization system, during the particle transfer from the bottom column into the depressurization tank, a small amount of compressed gas moves from the bottom column to the tank together with the particles. In order to return the atmospheric pressure in the bottom tank, this gas must be 
ejected to the outside and this is achieved by opening the valve F. The corresponding lost gas flow rate, $\dot{m}_{\text {lost }}^{D}$, can be expressed as follows:

$$
\dot{m}_{\text {lost }}^{D}=\frac{m_{\text {lost }}^{D}}{t_{c}^{D}}
$$

where $t_{c}^{D}$ denotes the duration of each depressurization cycle, and $m_{\text {lost }}^{D}$ is the mass of compressed gas lost in one depressurization cycle, as well as the mass of compressed gas which occupies the interstices of the particles within the bottom tank: this amount can be considered equal to the entire mass of compressed gas which would occupy the entire bottom tank, reduced by a coefficient $f$, which represents the ratio between the gas volume and the volume of the bottom tank. Therefore, with this hypothesis, Equation (8) can be written as:

$$
\dot{m}_{\text {lost }}^{D}=\frac{\rho_{g}^{i n} v_{t}^{D} f}{t_{c}^{D}}
$$

where $\rho_{g}^{i n}$ is the density of the inlet gas stream in the bottom column. The duration of each depressurization cycle, $t_{c}^{D}$, can be expressed as follows:

$$
t_{c}^{D}=2 t_{d}^{D}+t_{x}^{D}
$$

where $t_{d}^{D}$ is the time interval of the particle discharge, as well as the time interval of the filling phase, and $t_{x}^{D}$ amounts the time intervals spent for the depressurization phase and for the valves actuation. Analyzing the single terms of Equation (10) and Equation (4), $t_{d}^{D}$ results to be equal to $t_{d}^{P}$ if the valves of the depressurization system have the same size of those of the pressurization system, whereas $t_{x}^{D}$ is slightly minor than $t_{x}^{P}$, which also amounts the time interval spent for the pressurization phase. As a consequence, $t_{c}^{D}$ is slightly minor than $t_{c}^{P}$, and the amount $t_{c}^{P}-t_{c}^{D}$ depends on the duration of the pressurization phase.

The average mass flow rate of particles in the bottom column, $\overline{\dot{m}}_{p}$, can be also related to the bottom tank volume $V_{t}^{D}$ and to the cycle time $t_{c}^{D}$ as follows:

$$
\overline{\dot{m}}_{p}=\frac{\rho_{p} V_{t}^{D}}{t_{c}^{D}}
$$

Equalizing Equation (11) with Equation (5), one obtains the expression of the volume $V_{t}^{D}$, depending on the volume $V_{t}^{P}$, and on the ratio between the two cycle times:

$$
V_{t}^{D}=\frac{t_{c}^{D} V_{t}^{P}}{t_{c}^{P}}
$$

Substituting Equation (3) and Equation (9) into Equation (1), and using the formula of $V_{t}^{P}$ (Equation (7)) and $V_{t}^{D}$ (Equation (12)), one obtains the expression of $\dot{m}_{\text {lost }} / \dot{m}_{g}$

$$
\frac{\dot{m}_{l o s t}}{\dot{m}_{g}}=\frac{c_{p, g}\left(\rho_{g}^{o u t}+\rho_{g}^{\text {in }} f\right)}{c_{p} \rho_{p}}
$$

It is noteworthy that the denominator of Equation (13) is significantly larger than the numerator, since the intermediate medium must be characterized by 
large values of specific heat and density. Equation (13) can be expressed in terms of pressures and temperatures, as follows:

$$
\frac{\dot{m}_{\text {lost }}}{\dot{m}_{g}}=\frac{c_{p, g} p_{g}^{c}}{R c_{p} \rho_{p}}\left(\frac{1}{T_{g}^{\text {out }}}+\frac{f}{T_{g}^{\text {in }}}\right)
$$

where $p_{g}^{c}$ is the pressure in the bottom column (assumed constant), $T_{g}^{\text {in }}$ and $T_{g}^{\text {out }}$ are respectively the temperature of the gas in the inlet and in the outlet of the bottom column, and $R$ is the gas constant.

In a gas turbine plant, $T_{g}^{i n}$ depends on the pressure $p_{g}^{c}$ and on the characteristics of the axial compressor, namely on the isentropic efficiency $\eta_{i s, c}$, as follows:

$$
T_{g}^{i n}=T_{a t m}\left[1+\frac{{\frac{p_{g}^{c}}{p_{a t m}}}^{\frac{k-1}{k}}-1}{\eta_{i s, c}}\right]
$$

In Figure 2, $\dot{m}_{\text {lost }} / \dot{m}_{g}$ is plotted as a function of $T_{g}^{\text {out }}$, for three fixed values of $p_{g}^{c}$, namely 30 bar, 25 bar and 20 bar. The curves of $\dot{m}_{\text {lost }} / \dot{m}_{g}$ have been obtained by employing Equations (14) and (15), with the assumption that the intermediate medium is alumina. Once fixed $p_{g}^{c}$, Equation (15) has allowed us to compute $T_{g}^{i n}$, assuming $T_{a t m}=288 \mathrm{~K}, p_{a t m}=1$ bar and $\eta_{i s, c}=0.9$. The resulting value of $T_{g}^{i n}$ has been substituted into Equation (14) and $\dot{m}_{\text {lost }} / \dot{m}_{g}$ has been calculated as a function of $T_{g}^{\text {out }}$. Specifically, $T_{g}^{\text {out }}$ has been incremented from the minimum value, which has been set equal to the value of $T_{g}^{i n}$ (computed by Equation 15), to the maximum allowable value equal to $1500 \mathrm{~K}$. Clearly, the condition $T_{g}^{\text {out }}$ $=T_{g}^{i n}$ is only a mathematical assumption, since the bottom column must operate with an overall temperature gradient greater than zero. With regard to $f$, we have fixed this parameter equal to 0.5 , assuming that in the worst hypothesis the volume of the gas is equal to the volume of the particles within the bottom tank.

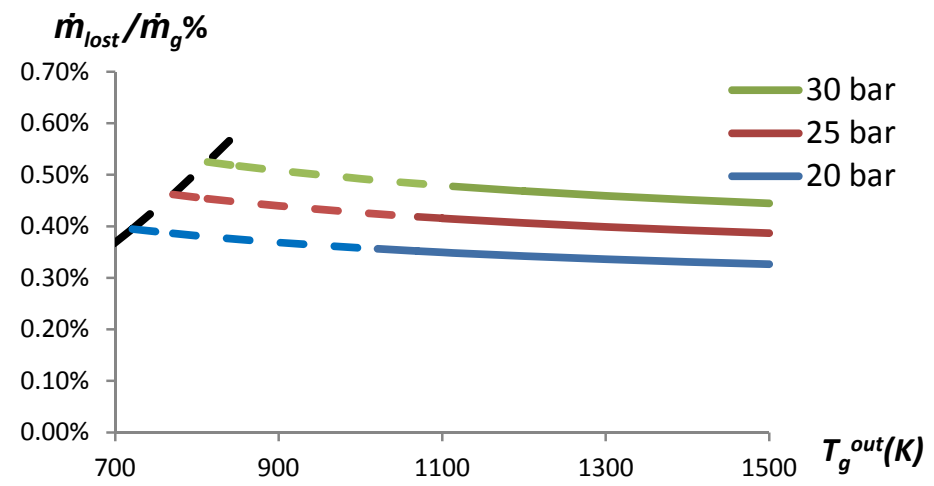

Figure 2: $\quad \dot{\mathrm{m}}_{\text {lost }} / \dot{\mathrm{m}}_{\mathrm{g}} \%$ versus $T_{g}^{\text {out }}$ for 3 values of $p_{g}^{c}$. 
In Figure 2, the black dotted line denotes the curve of $T_{g}^{i n}$, which only depends on $p_{g}^{c}$. Note that each curve at constant pressure is formed by two parts, namely a first part (dotted line) that occurs for small values of $T_{g}^{\text {out }}-T_{g}^{\text {in }}$, and a second one (continuous line) that occurs for large values of $T_{g}^{\text {out }}-T_{g}^{\text {in }}:$ we have fixed the demarcation point between the two parts equal to $T_{g}^{o u t}-T_{g}^{\text {in }}=300 \mathrm{~K}$. Therefore, only the continuous lines are representative of the real conditions occurring in typical turbine plants, which would require very large values of $T_{g}^{\text {out }}-T_{g}^{\text {in }}$. As shown by the trends of the graph, $\dot{m}_{\text {lost }} / \dot{m}_{g} \%$ increases both with decreasing $T_{g}^{\text {out }}$ and with increasing $p_{g}^{c}$, but the maximum value of $\dot{m}_{\text {lost }} / \dot{m}_{g} \%$ is under $0.55 \%$ in the worst condition.

\subsection{Cooling of the intermediate medium}

When the intermediate medium is contained in the pressurization tank, it mixes with the cold residual gas remained in the capacity at the end of the gas discharge phase. However, the cooling of the hot particles and the related energy loss can be neglected, due to the great difference between heat capacities of the intermediate medium and of the residual gas.

\subsection{Mechanical power required by the conveyor}

The Heat Exchanger must operate continuously in a real plant, therefore the particles, after having exited the bottom tank, must be delivered back to the top of the plant by using a conveyor. The power absorbed by the conveyor, $\mathrm{P}_{1}$, can be estimated with respect to the overall thermal power transferred from the particles to the gas in the bottom column, $\dot{Q}$. The ratio $\mathrm{P}_{1} / \dot{Q}$ can be expressed as:

$$
\frac{P_{l}}{\dot{Q}}=\frac{g \overline{\dot{m}}_{p} H}{g c_{p, g}\left(T_{g}^{\text {out }}-T_{g}^{\text {in }}\right)}
$$

where $g$ is the acceleration of gravity and $H$ is the overall height of the heat exchanger. The numerator and the denominator of Equation (16) represent the power adsorbed by the conveyor and the heating power released by the particles in the bottom column, respectively. With simple arguments, $\mathrm{P}_{1} / \dot{Q}$ results in:

$$
\frac{P_{l}}{\dot{Q}}=\frac{g H}{c_{p}\left(T_{g}^{\text {out }}-T_{g}^{\text {in }}\right)}
$$

In Figure 3, $\mathrm{P}_{1} / \dot{Q}$ is plotted as a function of $H$, for three values of $T_{g}^{\text {out }}-T_{g}^{\text {in }}$, with the assumption that the particle material is alumina $\left(c_{p}=1025 \mathrm{~J} /(\mathrm{Kg} \mathrm{K})\right)$. As shown by the trends of the graph, $\mathrm{P}_{1} / \dot{Q} \%$ increases both with decreasing $T_{g}^{\text {out }}-T_{g}^{\text {in }}$ and with increasing, but the maximum value of $\mathrm{P}_{1} / \dot{Q} \%$ is under $0.02 \%$. The energy loss due to the employment of the conveyor is absolutely negligible. 


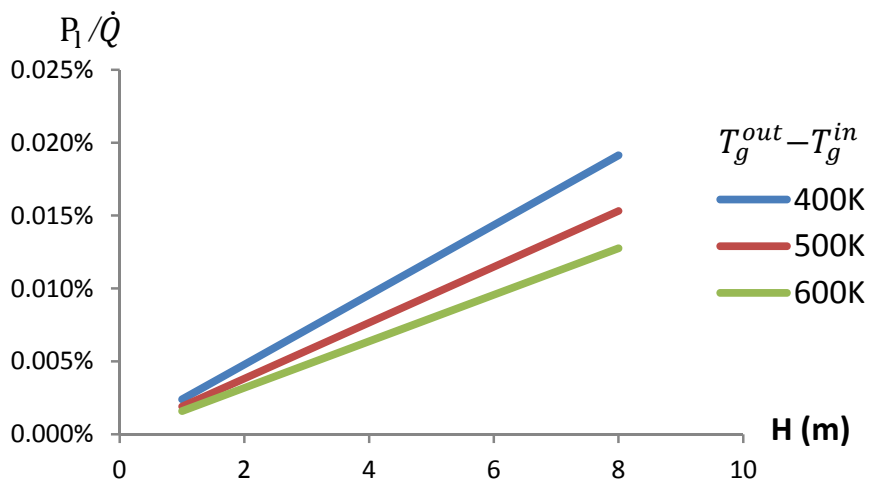

Figure 3: $\quad P_{l} / \dot{Q} \%$ versus $H$ for 3 values of $T_{g}^{o u t}-T_{g}^{\text {in }}$.

\section{Conclusions}

This paper has analysed the energy losses related to some key components of an immersed particle gas to gas heat exchanger, previously proposed by the authors and currently under full development. Such heat exchanger requires, for the application to gas turbine plants, a pressurization and a depressurization device, in order to transfer the solid particles between environments at different pressure. The paper has described the operating principles of the pressurization and depressurization procedures and the mechanical systems based on these procedures. Simple analytical models have been used to show that all energy losses related to the proposed mechanical systems are negligible, even in case of very high pressure values. Finally, the energy absorbed by the conveyor to move the particles from the bottom to the top of the plant has been evaluated with respect to the overall thermal energy exchanged between the particles and the gas in the bottom column: also this energy loss is absolutely negligible.

\section{Nomenclature}

$c_{p, g} \quad$ Constant pressure specific heat of gas $[\mathrm{J} /(\mathrm{Kg} \mathrm{K})]$

$c_{p} \quad$ Specific heat of particles $[\mathrm{J} /(\mathrm{Kg} \mathrm{K})]$

$c_{v, g} \quad$ Constant volume specific heat of gas $[\mathrm{J} /(\mathrm{Kg} \mathrm{K})]$

$f \quad$ Ratio between the gas volume and the volume of the bottom tank

$g \quad$ Acceleration of gravity $\left[\mathrm{m} / \mathrm{s}^{2}\right]$

$K \quad$ Ratio of the specifics heats of the gas

$H \quad$ Height of the heat exchanger [m]

$\dot{m}_{g} \quad$ Mass flow rate of gas flowing through the bottom column [kg/s]

$m_{\text {lost }}^{D} \quad$ Mass of lost compressed gas during one depressurization cycle[kg]

$m_{\text {lost }}^{P} \quad$ Mass of lost compressed gas during one pressurization cycle[kg]

$\dot{m}_{\text {lost }} \quad$ Overall mass flow rate of compressed gas discharged to the external environment $[\mathrm{kg} / \mathrm{s}]$ 
$\dot{m}_{\text {lost }}^{D}$ Mass flow rate of compressed gas discharged during one depressurization cycle $[\mathrm{kg} / \mathrm{s}]$

$\dot{m}_{\text {lost }}^{P}$ Mass flow rate of compressed gas discharged during one pressurization cycle $[\mathrm{kg} / \mathrm{s}]$

$\overline{\dot{m}}_{p} \quad$ Particle mass flow rate required for heating the compressed gas in the bottom column $[\mathrm{kg} / \mathrm{s}]$

$n^{P} \quad$ Number of pressurization cycles [cycles/sec or cycles $/ \mathrm{h}$ ]

$p_{\text {atm }} \quad$ Atmospheric pressure [bar]

$p_{g}^{c} \quad$ Pressure of the compressed gas in the bottom column[bar]

$P_{l} \quad$ Power absorbed by the conveyor [W]

$\dot{Q} \quad$ Thermal power transferred from the particles to the gas in the bottom column $[\mathrm{J} / \mathrm{s}]$

$R \quad$ Gas constant $[\mathrm{J} /(\mathrm{Kg} \mathrm{K})]$

$t_{c}^{D} \quad$ Time of one depressurization cycle[s]

$t_{c}^{P} \quad$ Time of one pressurization cycle[s]

$t_{d}^{D} \quad$ Duration of the particle discharge phase (from the depressurization tank into the conveyor) [s]

$t_{d}^{P} \quad$ Duration of the particle discharge phase (from the pressurization tank into the bottom column) [s]

$T_{\text {atm }} \quad$ Atmospheric temperature[K]

$T_{g}^{i n} \quad$ Temperature of the compressed gas in the inlet of the bottom column $[\mathrm{K}]$

$T_{g}^{\text {out }} \quad$ Temperature of the compressed gas in the outlet of the bottom column $[\mathrm{K}]$

$t_{x}{ }^{D} \quad$ Interval time occurring for depressurization and valve actuation (depressurization cycle) [s]

$t_{x}^{P} \quad$ Interval time occurring for pressurization, gas discharge and valve actuation (pressurization cycle) [s]

$V_{t}^{D} \quad$ Volume of the depressurization $\operatorname{tank}\left[\mathrm{m}^{3}\right]$

$V_{t}^{P} \quad$ Volume of the pressurization $\operatorname{tank}\left[\mathrm{m}^{3}\right]$

$\eta_{i s, c} \quad$ Isentropic efficiency of the compressor

$\rho_{g}^{i n} \quad$ Density of gas in the inlet of the bottom column $\left[\mathrm{kg} / \mathrm{m}^{3}\right]$

$\rho_{g}^{\text {out }} \quad$ Density of gas in the outlet of the bottom column $\left[\mathrm{kg} / \mathrm{m}^{3}\right]$

$\rho_{p} \quad$ Particle density $\left[\mathrm{kg} / \mathrm{m}^{3}\right]$

\section{References}

[1] Correa M. S., Power generation and aeropropulsion gas turbines: From combustion science to combustion technology. International Symposium on Combustion, Volume 27, Issue 2, 1998, Pages 1793-1807.

[2] McDonald, C. F. e Wilson, D. G., The utilization of recuperated and regenerated turbine engine cycles for high-efficiency gas turbine in 21ct century, Applied Thermal Engineering, 1995, Vol. 16, No. 8-9, pp. 635-653. 
[3] Rolls Royce Group plc., Annual Report and Accounts, 2008, available at www.rolls-royce.com/reports/2008.

[4] Dallenback, P.A., Improved gas turbine efficiency through alternative regenerator configuration, ASME, Journal of Gas Turbine and Power, 2002.,Vol. 124, pp. 441-446.

[5] Zimmermann, P., Cardenas, A., Hirsch, C., and Sattlemayer, T., Simulation of a Micro Turbine's Dynamic Behavior in a Biomass Incineration Power Plant Based on the Pebble Heater Technology, ASME Paper GT200959305, Orlando, FL, USA, 2009.

[6] Brzozowski, W., Dul, J., Yerouchalmi, D., and Jadrowitch, B., Pebble Bed Heat Exchanger, U.S. Patent Office, 1968, U.S. Patent No. 3488402.

[7] Theoclitus, G., Pebble Bed Heat Exchanger, Canadian Intellectual Property Office, 1958, CA 557552D.

[8] Catalano L. A., De Bellis F., Amirante R., Rignanese M., An Immersed Particle Heat Exchanger for Externally Fired and Heat Recovery Gas Turbines, Journal of Engineering for Gas Turbine and Power, March 2011, Vol. 133, Issue 3, 032301 (7 pages).

[9] F. De Bellis, L. A. Catalano, "CFD Optimization of an Immersed Particles Heat Exchanger", Applied Energy, 2012, to appear.

[10] L. Nettis, F. De Bellis, L. Catalano, R. Verzicco, "Unsteady Conjugate Heat Transfer Analysis of an Immersed Particle Innovative Heat Exchanger", Journal of Thermal Science and Engineering Applications, 2012, to appear.

[11] Hilton J. E., Mason L. R., Cleary P. W., The effect of gas dynamics on pressurization tank discharge rate, CSIRO Mathematical and Information Sciences, Clayton, Victoria, Australia, 9-11 December 2009. 